\title{
Endoscopic deployment of multiple $(\geq 3)$ metal stents for unresectable malignant hilar biliary strictures $\square$
}

\section{다 (1) $\odot$}

Authors

Tatsuya Koshitani ${ }^{1}$, Shuji Nakagawa ${ }^{1,2}$, Yoshitomo Konaka ${ }^{1}$, Keimei Nakano ${ }^{1}$, Shuichi Fuki ${ }^{1}$, Yoshito Itoh ${ }^{3}$

Institutions

1 Department of Gastroenterology, Japan Community Health Care Organization Kobe Central Hospital, Kobe, Japan

2 Nakagawa Naika Clinic, Kobe, Japan

3 Department of Molecular Gastroenterology and Hepatology, Kyoto Prefectural University of Medicine, Graduate School of Medical Science, Kyoto, Japan

submitted 16.8.2018

accepted after revision 3.12 .2018

Bibliography

DOI https://doi.org/10.1055/a-0849-9334 |

Endoscopy International Open 2019; 07: E672-E677

(c) Georg Thieme Verlag KG Stuttgart · New York elSSN 2196-9736

Corresponding author

Tatsuya Koshitani, MD, PhD, Department of

Gastroenterology, Japan Community Health Care

Organization Kobe Central Hospital, 2-1-1 Soyamacho

Kita-ku, Kobe, 6511145, Japan

Fax: +81-78-594-2244

tkoshitani@aol.com

\section{ABSTRACT}

Background and study aims Endoscopic deployment of multiple $(\geq 3)$ self-expandable metal stents (SEMS) for high-grade unresectable malignant hilar biliary strictures (UMHBS) is technically challenging. Eleven consecutive patients with high-grade UMHBS (mean age: 76 years, male/ female: 5/6, Bismuth-Corlette classification IIla/IV: 7/4) underwent endoscopic deployment of multiple SEMS using a combination of side-by-side (SBS) and stent-in-stent (SIS) methods. Technical and clinical success rates were 11/11. More than three SEMS were successfully deployed, and obstructive jaundice was fully improved in all cases. Stent occlusion was recognized in four of 11 patients (mean: 134 days, range: 28 -232). Reinterventions for both liver lobes were feasible by passing the guide wire inside the previously placed stents in three of four patients. Median stent patency was 150 days during a mean follow-up period of 184 days (range: $37-558)$. Three patients developed self-limiting cholangitis without definite stent occlusion as late (>30 days) adverse events. Employing the combination of SBS and SIS methods may facilitate endoscopic deployment of multiple SEMS to treat high-grade UMHBS.

\section{Introduction}

For patients with unresectable malignant hilar biliary strictures (UMHBS), palliative biliary drainage is the treatment of choice. This can be performed surgically, percutaneously, or endoscopically. Although endoscopic transpapillary biliary drainage is the preferred method because of its minimal invasiveness, ongoing controversy exists in the selection of appropriate devices, the range of biliary drainage, and the method of stent deployment.

There are two methods of deploying bilateral self-expandable metal stents (SEMS) endoscopically: side-by-side (SBS) and stent-in-stent (SIS). Using the SBS method, two SEMS are deployed in the right and left hepatic ducts parallel to each other, enabling selective reintervention. On the other hand, using the SIS method, the second SEMS is deployed in the contralateral hepatic duct through the mesh of the first SEMS. This enables stent placement that is fitted to the bile duct structure and reduces risk of excessive compression in the hilum. To date, there have been only two retrospective studies comparing outcomes between SBS and SIS methods, and the results have been controversial [1,2].

Endoscopic deployment of multiple SEMS ( $\geq 3)$ using the SIS method was reported to treat high-grade UMHBS $[3,4]$. This treatment was performed as a reasonable option to control cholangitis and preserve the functional volume of the liver as much as possible prior to chemotherapy. The idea is very attractive; however, problems remain regarding technical difficulties with initial stent deployment and reintervention for stent occlusion. To overcome these problems, we deployed multiple SEMS using a combination of SBS and SIS methods to treat high-grade UMHBS [5]. In this study, we reviewed a series of the cases treated with this technique and clarified its usefulness. 


\section{Case reports}

The study was approved by the ethical committee of Japan Community Health Care Organization Kobe Central Hospital and registered in University Hospital Medical Information Network Clinical Trials Registry (UMIN000033685).

From August 2015 to March 2018, 11 consecutive patients with high-grade UMHBS (mean age: 76 years, range: 53-88 years, male/female: 5/6, Bismuth-Corlette classification IIla/IV: $7 / 4)$ underwent endoscopic deployment of multiple SEMS $(\geq 3)$ using a combination of SBS and SIS methods. The cases involved six cholangiocarcinomas, three gallbladder carcinomas, one cholangiocellular carcinoma, and one hepatocellular carcinoma. Pathology was confirmed cytologically and/or histologically in all patients preoperatively. The patients' characteristics are shown in $>$ Table 1.

\section{SEMS deployment}

After initial drainage with endoscopic biliary stenting and/or endoscopic nasobiliary drainage, SEMS were typically deployed as follows. After selective cannulation using a tapered-tip catheter (MTW Endoskopie, Wesel, Germany) and a 0.025-inch guide wire (VisiGlide 2; Olympus Medical Systems, Tokyo, Japan), two 6-Fr stent delivery systems (Zilver 635 Biliary Self-Expanding Stent, 8-mm stent diameter; Cook Medical, Tokyo, Japan) were simultaneously inserted through the working channel of a therapeutic duodenoscope (TJF-260V; Olympus Medical Systems, Tokyo, Japan). They were positioned such that one was in the right posterior sectoral duct and one in the left hepatic duct. The SEMS were deployed using the SBS method with the distal stent markers aligned within the duct to facilitate selective reintervention. Next, a 0.025 -inch guide wire was introduced into the right anterior sectoral duct through the mesh of the SEMS on the right side. Then, the mesh of the stent was dilated with a 6-mm balloon (REN; Kaneka Medix, Osaka, Japan), the guide wire was exchanged for a 0.035-inch stiff guide wire (Wrangler; Piolax, Kanagawa, Japan), and the delivery system was introduced. Finally, another SEMS was deployed in the right anterior sectoral duct using the SIS method ( $\triangleright$ Fig.1a, $>$ Fig.1b, $>$ Fig.1c, $>$ Fig.1d, $\triangleright$ Fig.1e, $\triangleright$ Fig.1f, - Video 1).

\section{Outcome measurements}

We retrospectively reviewed the following outcomes: 1 ) technical and clinical success rates; 2 ) reinterventions; 3 ) stent patency; and 4 ) early ( $\leq 30$ days) and late (>30 days) adverse events (AEs). Technical success was defined as successful deployment of multiple SEMS $(\geq 3)$ at the intended position. Clinical success was defined as a decrease of more than $50 \%$ in serum total bilirubin (T-Bil) level within 1 month after SEMS deployment compared with the pre-intervention level [6]. Stent occlusion was defined as recurrence of biliary obstruction and jaundice and/ or evidence of cholestasis confirmed by computed tomography, requiring biliary reintervention. Reinterventions were evaluated by type of treatment and feasibility of treatment for both liver lobes. Stent patency was defined as the period between SEMS deployment and stent occlusion. Any death before
- Table 1 Patient characteristics.

\begin{tabular}{|l|l|}
\hline & Data \\
\hline Number of patients & 11 \\
\hline Age (yr), mean & 76 (range 53-88) \\
\hline Gender (n, \%) & \\
\hline - Male & $5,45.5$ \\
\hline - Female & $6,54.5$ \\
\hline Diagnosis (n, \%) & \\
\hline - Cholangiocarcinoma & 6,545 \\
\hline - Gall bladder carcinoma & $3,27.3$ \\
\hline - Cholangiocellular carcinoma & $1,9.1$ \\
\hline - Hepatocellular carcinoma & $1,9.1$ \\
\hline Type of stenosis ${ }^{1}$ (n, \%) & \\
\hline - Illa & $7,63.6$ \\
\hline - IV & $4,36.4$ \\
\hline T-Bil (mg/dL), mean & 15 (range 2-27) \\
\hline ALP (U/L), mean & 1738 (range 554-2687) \\
\hline Follow-up period (days), mean & 184 (range 37-558) \\
\hline T-Bil, total bilirubin; ALP, alkaline phosphatase. \\
\hline Bismuth-Corlette classification & \\
\hline
\end{tabular}

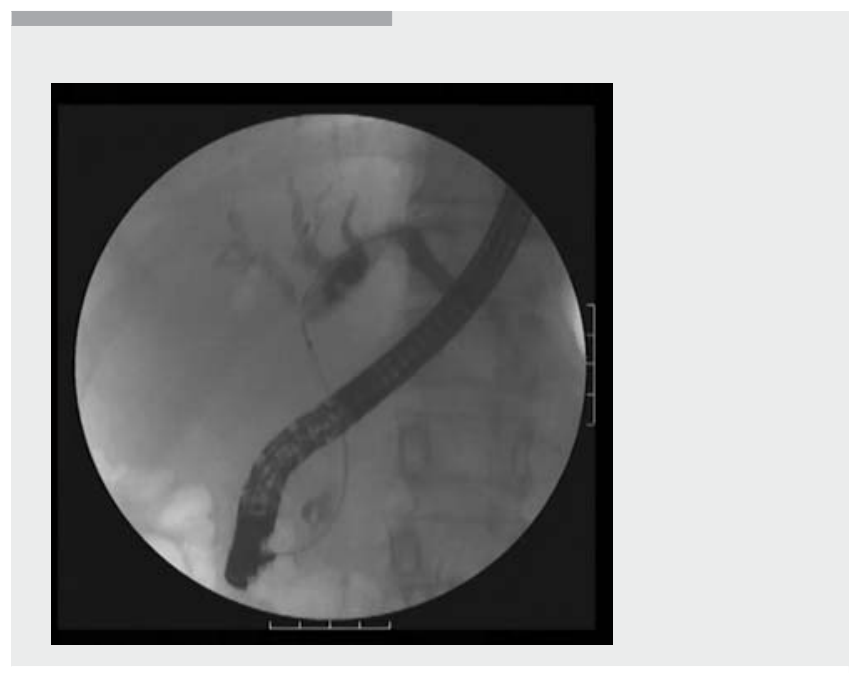

$\triangle$ Video 1 After selective cannulation using a 0.025 -inch guide wire, the SEMS were deployed in the right posterior sectoral duct and the left hepatic duct using the SBS method. Next, a 0.025 -inch guide wire was introduced into the right anterior sectoral duct through the mesh of the SEMS on the right side. Then, the mesh of the stent was dilated with a $6-\mathrm{mm}$ balloon, the guide wire was exchanged for a 0.035 -inch stiff guide wire, and the delivery system was introduced. Finally, another SEMS was deployed in the right anterior sectoral duct using the SIS method. 

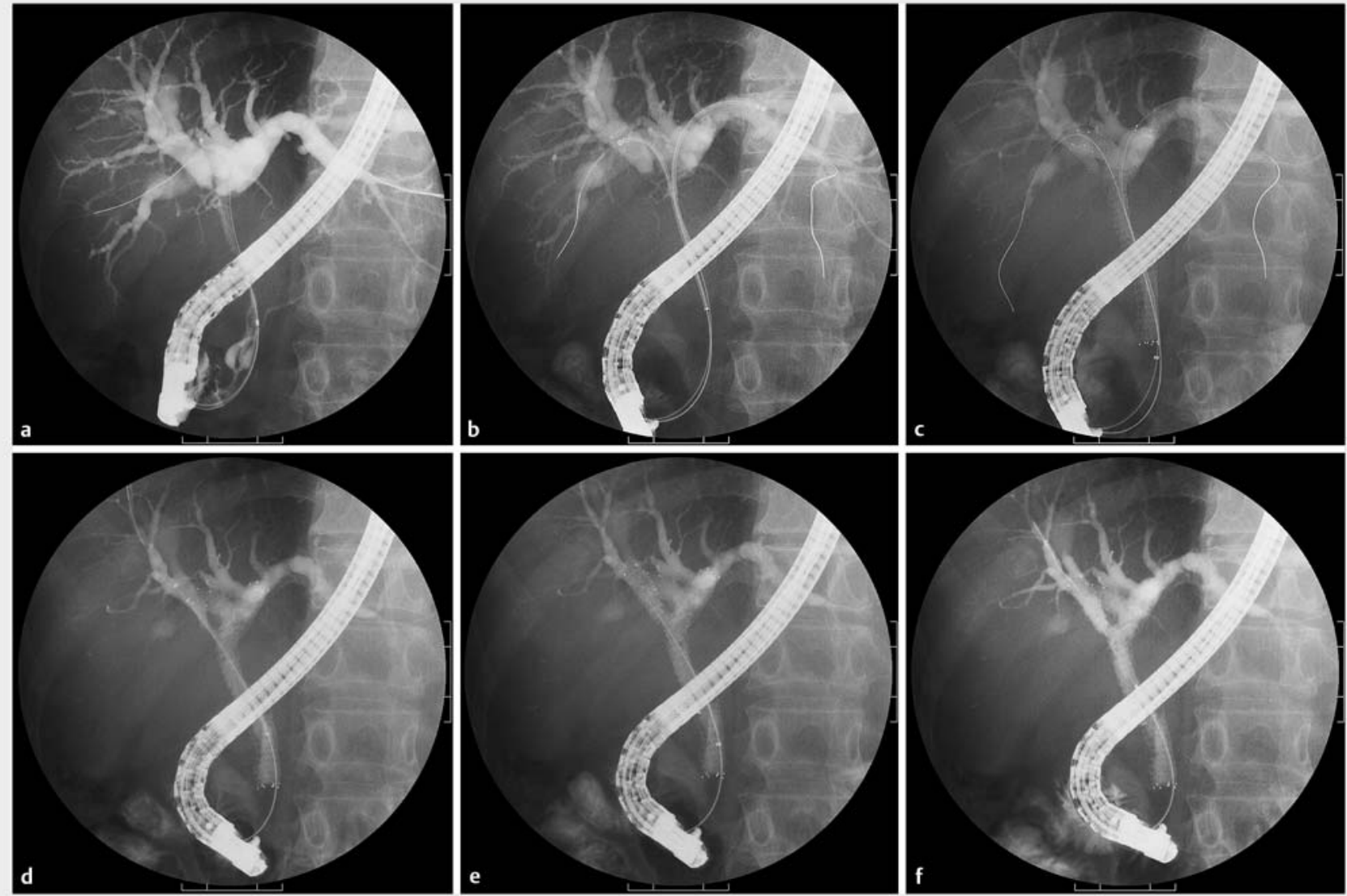

Fig. 1 a, b, c After selective cannulation using a 0.025-inch guide wire, the SEMS were deployed in the right posterior sectoral duct and the left hepatic duct using the SBS method. $\mathbf{d}$ After balloon dilation and exchange for a 0.035 -inch stiff guide wire, the stent delivery system was introduced into the right anterior sectoral duct through the mesh of the SEMS on the right side. e, $\mathbf{f}$ Another SEMS was deployed in the right anterior sectoral duct using the SIS method.

stent occlusion was treated as censored data when calculating stent patency. Follow-up was performed until May 1, 2018.

\section{Statistical analysis}

Continuous variables were described using mean and range, and categorical variables were expressed as proportions. Wilcoxon signed-rank test was used to compare laboratory parameters before and after deploying SEMS. A $P$ value <.05 was considered statistically significant. Stent patency was analyzed by the Kaplan-Meier method.

\section{Demographic data and outcomes}

Demographic data on and outcomes for each patient are shown in $>$ Table 2. The technical success rate was $11 / 11$. More than three SEMS were successfully deployed at the intended position in all cases. The clinical success rate was also $11 / 11$. The mean serum T-Bil level decreased significantly 1 month after deploying SEMS, and there was no exacerbation in any patients after deploying SEMS. Stent occlusion was recognized in four patients (mean: 134 days, range: 28 -232). Causes of stent occlusion were tumor ingrowth in two cases, tumor overgrowth in one, and biliary sludge in one. Tumor ingrowth and overgrowth were treated by additional deployment of SEMS or plastic stent (PS). Biliary sludge inside the stents was eliminated by balloon retrieval. Reinterventions for both liver lobes were feasible by passing the guide wire inside the previously placed stents in

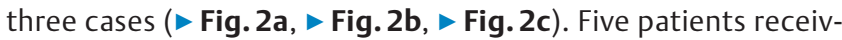
ed additional chemotherapy (gemcitabine plus cisplatin). Median stent patency was 150 days during a mean follow-up period of 184 days (range: 37-558; > Fig.3). Three patients developed self-limiting cholangitis without definite stent occlusion as late (>30 days) AEs. They were managed with conservative therapy ( $>$ Table 3 ).

\section{Discussion}

Several studies have highlighted the advantages of SEMS compared with PS, and recent meta-analyses have shown that SEMS are associated with significantly higher successful drainage, fewer complications, longer stent patency, and longer patient survival in malignant hilar lesions [6,7].

Debate is ongoing regarding the range of drainage in cases with UMHBS [6]. The reason to support bilateral stent placement is based on the concept that draining a volume greater 
- Table2 Demographic data on and outcome for each patient.

\begin{tabular}{|c|c|c|c|c|c|c|c|c|c|c|}
\hline \multirow{2}{*}{$\begin{array}{l}\text { Patient } \\
\text { no. }\end{array}$} & \multirow{2}{*}{$\begin{array}{l}\text { Age } \\
\text { (yr) }\end{array}$} & \multirow[t]{2}{*}{ Gender } & \multirow{2}{*}{$\begin{array}{l}\text { Diagno- } \\
\text { sis }\end{array}$} & \multirow{2}{*}{$\begin{array}{l}\text { Type of } \\
\text { stenosis }^{1}\end{array}$} & \multirow{2}{*}{$\begin{array}{l}\text { Initial drainage } \\
\text { (No. of stents) }\end{array}$} & \multirow{2}{*}{$\begin{array}{l}\text { Method } \\
\text { (No. of stents) }\end{array}$} & \multicolumn{2}{|c|}{ Stent patency } & \multicolumn{2}{|c|}{ Survival } \\
\hline & & & & & & & Days & Status & Days & Status \\
\hline 1 & 84 & $\mathrm{~F}$ & $\mathrm{CC}$ & Illa & ENBD (1) & SBS + SIS (3) & 150 & Obstructed & 447 & Dead \\
\hline 2 & 85 & $\mathrm{~F}$ & $\mathrm{CC}$ & IV & EBS (2) & $S B S+S I S(3)$ & 392 & Patent & 392 & Dead \\
\hline 3 & 74 & M & CC & IV & $\begin{array}{l}\operatorname{EBS}(1) \\
\operatorname{ENBD}(1)\end{array}$ & $S B S+S I S(3)$ & 103 & Patent & 103 & Dead \\
\hline 4 & 66 & $\mathrm{~F}$ & $\mathrm{CC}$ & Illa & $\begin{array}{l}\operatorname{EBS}(1) \\
\operatorname{ENBD}(1)\end{array}$ & $S B S+S I S(3)$ & 232 & Obstructed & 558 & Alive \\
\hline 5 & 79 & $\mathrm{~F}$ & $\mathrm{CC}$ & IV & EBS (2) & $S B S+S I S(4)$ & 124 & Obstructed & 143 & Dead \\
\hline 6 & 53 & M & $\mathrm{CC}$ & Illa & $\operatorname{EBS}(1)$ & $S B S+S I S(3)$ & 54 & Patent & 54 & Alive \\
\hline 7 & 80 & M & GBC & IIla & $\operatorname{ENBD}(1)$ & SBS + SIS (3) & 70 & Patent & 70 & Dead \\
\hline 8 & 65 & $\mathrm{~F}$ & GBC & Illa & $\operatorname{EBS}(2)$ & $S B S+S I S(3)$ & 104 & Patent & 104 & Dead \\
\hline 9 & 88 & $\mathrm{~F}$ & GBC & Illa & $\operatorname{EBS}(2)$ & $S B S+S I S(3)$ & 28 & Obstructed & 69 & Dead \\
\hline 10 & 75 & M & $\mathrm{CCC}$ & IV & $\begin{array}{l}\operatorname{EBS}(1) \\
\operatorname{ENBD}(1)\end{array}$ & SBS + SIS (3) & 43 & Patent & 43 & Dead \\
\hline 11 & 82 & M & $\mathrm{HCC}$ & Illa & EBS (3) & $S B S+S I S(4)$ & 37 & Patent & 37 & Dead \\
\hline
\end{tabular}

F, female; M, male; CC, cholangiocarcinoma; ENBD, endoscopic nasobiliary drainage; SBS, side-by-side; SIS, stent-in-stent; EBS, endoscopic biliary stenting; GBC, gall bladder carcinoma; CCC, cholangiocellular carcinoma; HCC, hepatocellular carcinoma.

${ }^{1}$ Bismuth-Corlette classification
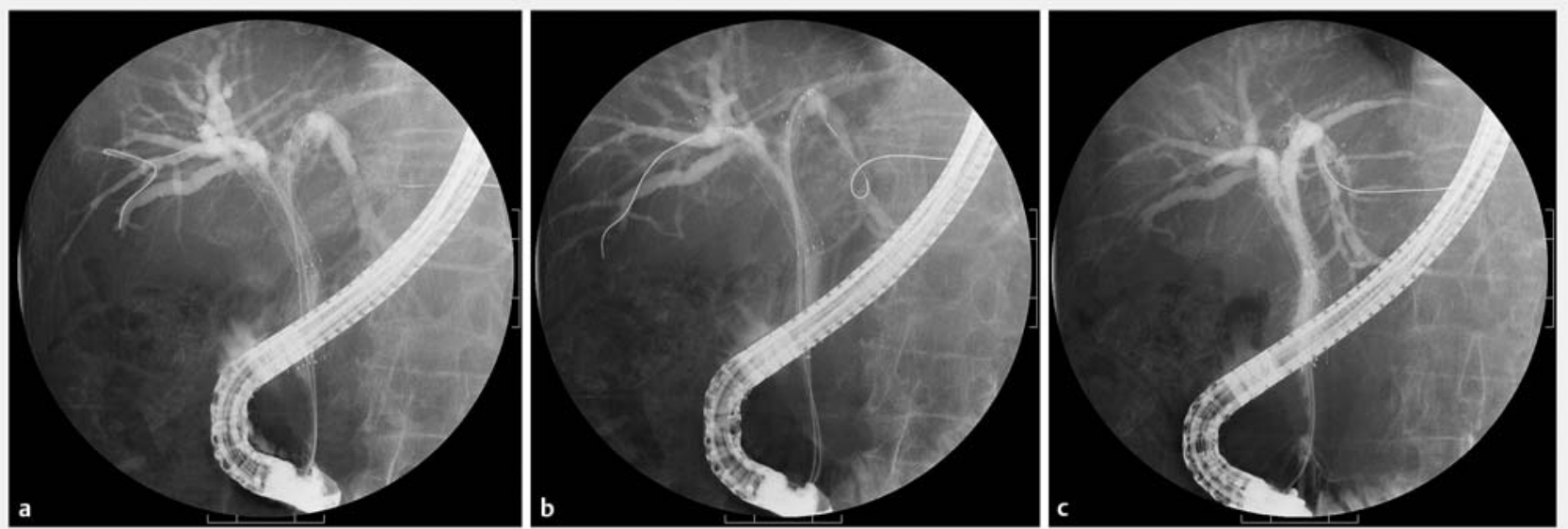

Fig. 2 Reintervention for both liver lobes. a 0.025 -inch guide wires were passed through the previously placed stents to both liver lobes. b, $\mathbf{c}$ Two additional SEMS were deployed inside the stents.

than $50 \%$ of the liver is associated with effective drainage and prolonged survival [8]. In a recent randomized controlled trial comparing outcomes of bilateral and unilateral placement of SEMS for UMHBS, both procedures had similar technical success rates, but bilateral drainage resulted in fewer reinterventions and more durable stent patency [9].

Regarding treatment for high-grade UMHBS, Kawamoto et al. [3] reported endoscopic three-branched partial SIS deployment of SEMS. In this method, two additional SEMS were deployed in the right anterior and posterior sectoral ducts through the mesh of the SEMS first placed in the left hepatic duct. Similarly, as a revisionary method, an additional third metal stent was deployed into a bilateral SIS configuration with cross-wired metal stents using this technique [4].

With the advent of the 6-Fr delivery system, we deployed multiple SEMS endoscopically using a combination of the SBS and SIS method to treat high-grade UMHBS. This technique has the following advantages. First, it may be easy to deploy the third stent. Using this method, the stent delivery system passes through the mesh of only one stent to deploy the third stent. Second, alignment of the distal stent edges using the SBS method may permit passage of the guide wire through the 


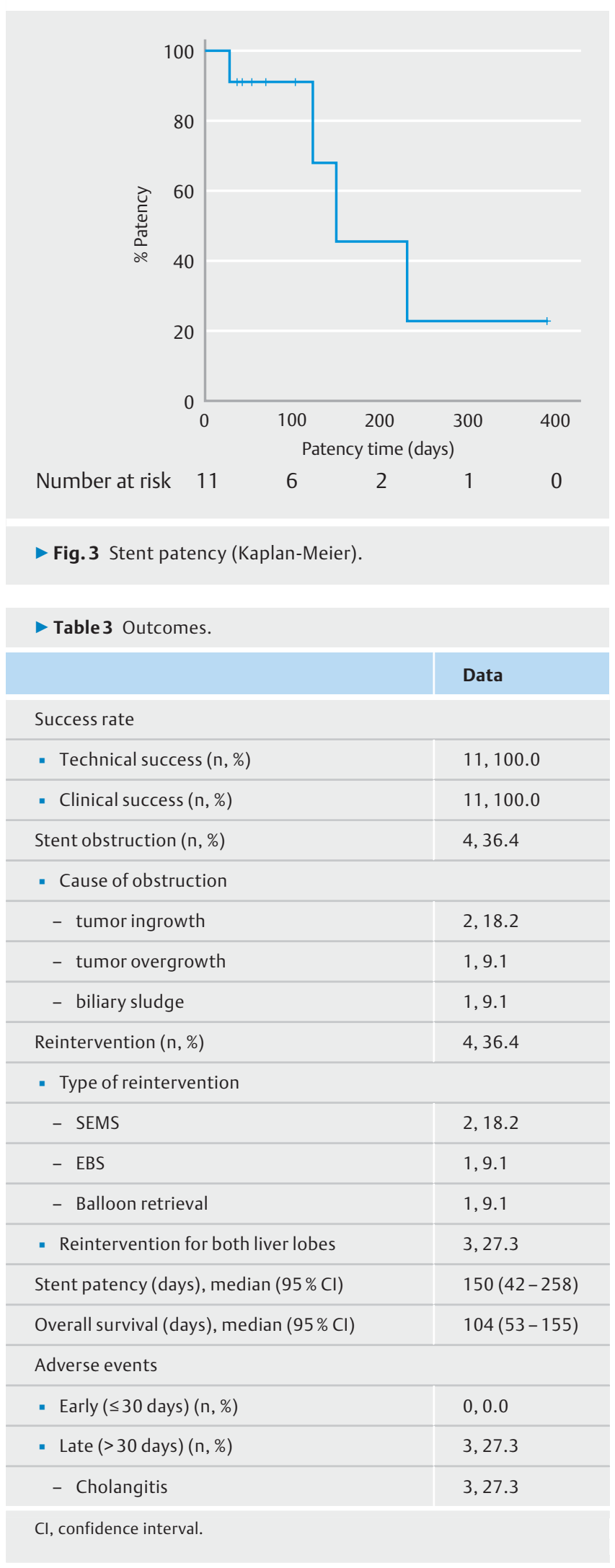

stents to both liver lobes for selective reintervention. Although reinterventions for both liver lobes were feasible in three of four cases in our study, they are still difficult after SBS stent placement above the papilla using laser-cut SEMS. Actually, we failed to pass a guide wire through the occluded stent to the left lobe in one case. It might be helpful to align the distal stent edges in the duodenum for future access to reintervention.

Median stent patency was 150 days in our study. We used 8$\mathrm{mm}$ metal stents. Compared with recent reports, stent patency in this study may be short. We speculate that stent patency might be underestimated because our cases included patients with poor prognosis and serious comorbidities and any death before stent occlusion was treated as censored data.

This study is limited by the small number of cases in a single center. The noncomparative nature of this study is also a limitation. Furthermore, whether SBS or SIS is the preferred method for bilateral SEMS remains unclear. Nevertheless, high technical and clinical success rates, low incidence of AEs, and good recovery from stent occlusion were observed in this study. We expect that the procedure shown in this study will become an option for treating high-grade UMHBS.

\section{Conclusion}

In conclusion, it is speculated that some cases with high-grade UMHBS require placement of multiple stents $(\geq 3)$ to control jaundice and cholangitis. Employing the combination of SBS and SIS methods may facilitate the endoscopic deployment of multiple SEMS for such cases.

\section{Competing interests}

None

\section{References}

[1] Naitoh I, Hayashi K, Nakazawa T et al. Side-by-side versus stent-instent deployment in bilateral endoscopic metal stenting for malignant hilar biliary obstruction. Dig Dis Sci 2012; 57: 3279-3285

[2] Kim KM, Lee KH, Chung YH et al. A comparison of bilateral stenting methods for malignant hilar biliary obstruction. Hepatogastroenterology 2012; 59: $341-346$

[3] Kawamoto H, Tsutsumi K, Fujii M et al. Endoscopic 3-branched partial stent-in-stent deployment of metallic stents in high-grade malignant hilar biliary stricture (with videos). Gastrointest Endosc 2007; 66: $1030-1037$

[4] Lee TH, Moon JH, Choi H] et al. Third metal stent for revision of malignant hilar biliary strictures. Endoscopy 2016; 48: 1129-1133

[5] Koshitani T, Nakagawa S, Itoh Y. Multiple self-expandable metal stent deployment for unresectable malignant hilar biliary strictures: combination of side-by-side and stent-in-stent methods. Dig Endosc 2016; 28: 621

[6] Isayama H, Hamada T, Yasuda I et al. Tokyo Criteria 2014 for transpapillary biliary stenting. Dig Endosc 2015; 27: 259-264

[7] Sawas T, Al Halabi S, Parsi MA et al. Self-expandable metal stents versus plastic stents for malignant biliary obstruction: a meta-analysis. Gastrointest Endosc 2015; 82: 256-267 
[8] Hong W, Sun X, Zhu Q. Endoscopic stenting for malignant hilar biliary obstruction: should it be metal or plastic and unilateral or bilateral? Eur J Gastroenterol Hepatol 2013; 25: 1105-1112

[9] Vienne A, Hobeika E, Gouya $\mathrm{H}$ et al. Prediction of drainage effectiveness during endoscopic stenting of malignant hilar strictures: the role of liver volume assessment. Gastrointest Endosc 2010; 72: 728 - 735
[10] Lee TH, Kim TH, Moon JH et al. Bilateral versus unilateral placement of metal stents for inoperable high-grade malignant hilar biliary strictures: a multicenter, prospective, randomized study (with video). Gastrointest Endosc 2017; 86: 817-827 